\title{
Superinfection Immunity
}

BG Blasdel and ST Abedon, The Ohio State University, Mansfield, OH, USA

(c) 2013 Elsevier Inc. All rights reserved.

This article is a revision of the previous edition article by E Kutter, volume 4, pp 1895-1896, () 2001, Elsevier Inc.

\section{Glossary}

cI The phage lambda $(\lambda)$ repressor $r_{i}$ it is necessary for maintaining lysogeny and acts by inhibiting, that is, repressing the transcription of phage genes required for the display of lytic eycles.

Homoimmunity Describes the relationship between two temperate phages hose lysegens are immune to superinfection by the other's phage particles, in contrast with heteroimmunity where temperate phages are not muttally immune.

Immunity (or superinfection immunity) Đisplay of homoimmunity against superinfecting phage particles. Lysogen A bacterium harbering a phage genome, as a prophage, that capable of a productive infection given induction.

Lysogenic cycle (or in 2 , An infection, by a temperate phage, that is associated with the formation of a bacterial lysogen and continued maintenance of a prophage, $\Omega$
Productive infection $A$ phage infection that results in virion production and either lytic or chronic virion release; most-productive infections are lytic, resulting in host lysis.

Prophage An intracellular phage genome stably associated with a bacterium during a lysogenic infection; often but not always found integrated into the host chromosome. Temperate phage A phage capable of displaying lysogenic as well as productive infections; most temperate phages have lytic rather than chronic productive infections and, following phage adsorption, productive rather than lysogenic infections often predominate.

Secondary adsorption Phage adsorption of an already phage-infected bacterium; depending upon circumstances this may or may not result in superinfection.

Superinfection Entrance by a phage into the cytoplasm of an already phage-infected bacterium; depending on circumstances this may or may not lead to lysogenic or ytiç infections.

\section{Introduction}

Phage life cycles can be distinguished into two main types, productive versus lysogenic. Unlike productive infections, in lysogeny phages produce no virions and instead are maintained as intracellular prophages that are able to block infections by similar phages. This occurs via the same mechanism that stabilizes existing lysogenic cycles: transcriptional repression of those phage genes required for the productive infection. The inhibitory impact of prophage-produced transcriptional repressor proteins on subsequently infecting phages can be described as an immunity to superinfection, superinfection immunity, or simply immunity. Superinfection immunity has been most thoroughly characterized in phage $\lambda$.

\section{Lysogeny and the CI Repressor}

During lysogeny, a prophage must prevent the transcription of genes whose expression can result in the induction of productive infections. In phage $\lambda$, the protein responsible for this transcriptional repression is encoded by the $c I$ gene and the product of the $c I$ gene, the CI protein, is commonly described as the $\lambda$ repressor.

Establishment of lysogeny involves what is known as a lysis-lysogeny decision, wh (1) pccurs 10-15 min after the start of infection with phas under standard laboratory conditions. Lysogenic cycles result when levels of $\lambda$ CII protein are high, whereas lytic cycles occur when levels of CII protein are low. CII protein, in particular, stimulates the expression of the $c I$ gene by activating a promoter, $P_{\mathrm{RE}}$, that control 1 ne transcription. As long as sufficient CI protein is present lysogeny will be both established and maintained. Once lysogeny has been established, CI protein stimulates the production of additional CI protein; in this case by acting on an additional $c I$ promoter, $P_{\mathrm{RM}}$. By also inhibiting $c I$ expression at higher $\mathrm{CI}$ concentrations, relatively constant levels of CI protein are maintained within $\lambda$ lysogens under normal growth conditions.

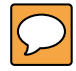

\section{Superinfection Im}

The $C$ epressor not prophage also hom nune phages happen to infect the same eell. The r. is a blocking ot Infection progression, that is, a display of sun fection immunity. Wild-type temperate phages that fail to respond in this manner are described instead as heteroimmune. Heteroimmune phages are not only capable of productively infecting established lysogens but are also able to lysogenically infect those same cells, resulting in polylysogeny. Polylysogeny may also result given superinfection by homoimmune phages if integrase genes, required for prophage establishment, are not controlled by the resident prophage's repressor.

Mutations in various phage genes can result in failure by otherwise temperate phages to produce lysogenic cycles. Mutations in the phage $\lambda c I$ gene can result in complete loss of an ability to lysogenize. Mutations in other $\lambda$ genes, particularly cII and cIII, too can result in substantial loss of that ability. Plaques are localized clearings of turbid bacterial cultures growing in or on agar-based media and these $\lambda$ phage mutants form 
clear plaques when plated on lawns of either non-lysogens or non-homoimmune lysogens. Plaques formed by wild-type temperate phages, by contrast, are turbid due to the establishment of phage-resistant lysogens over the course of plaque formation.

Phage $\lambda c I$, cII, and cIII mutants are still subject to superinfection immunity. Only certain double mutants in the $\lambda$ operator sequences, $O_{\mathrm{L}}$ and $O_{\mathrm{R}}$, which normally are bound by $\mathrm{CI}$ protein toward inhibition of phage transcription, are able to bypass superinfection immunity. As a result, these double mutants not only form clear plaques but do so even on lawns consisting of otherwise homoimmune lysogens.

The fact that it requires a double mutation for a homoimmune phage to bypass superinfection immunity pe strongest evidence that superinfection immunity pr es prophages with a selective utility. Such evidence is only circumstantial, however, and it is entirely possible that the only true benefit of superinfection immunity is protection from that subset of phages that are neither clonally related neteroimmune. It is an open question, in particular, whether blocking productive infections by clonally related, superinfecting phages would provide an adaptive utility to immunity from superinfection.

\section{Analogy, Practioality, and Conclusionc}

Phages are known to encode other mechanisms of resistance ta phages besides superinfection immunity. Among these mechanisms is the somewhat analogous superinfection exclusion. Superinfection exclusion displayed by the nontemperate phage $\mathrm{T} 4$, as associated with the expression of two genes, 'immunity' and 'spackle', is a means by which superinfection itself is prevented following secondary phage adsorption. That is, superinfection exclusion is a postattachment block on phage-genome translocation into the bacterial cytoplasm, whereas superinfection immunity's impact occurs after DNA translocation. Similar to superinfection immunity, it is uncertain whether superinfection exclusion's primary role is one of protection from subsequent phage infection since not only is that protection limited in breadth but also the gene products involved play important roles, at least with phage $\mathrm{T} 4$, in other aspects of phage infection.

Our general concl (51) is that both superinfection immunity and superinfectionchclusion may indeed play ecological roles in protecting phage infections from the disruption of superinfection, but evidence that these roles provide a selective benefit to the expressing prophages has not been robustly developed. Temperate phages, by displaying superinfection immunity, nevertheless, will tend to generate phage-resistant bacteria during their normal course of infection. That unquestionably is a problem given efforts to eliminate bacteria from environments using phages, that is, in the guise of what is known as phage therapy. For this as well as other reasons stemming from lysogen formation, the use of temperate phages is not recommended for phage therapy purposes.

Pecondary adsorption is to an already phage-infected Dacterium, such as of an already existing prophage. Superinfection exclusion blocks the initiation of superinfection by preventing phage entrance into the cytoplasm. Infection by homoimmune phages is blocked by superinfection immunity following phage-genome entrance into the cytoplasm. Heteroimmune superinfecting phages can successfully proceed with their infections, either productively or lysogenically, the latter resulting in polylysogens. The CI repressor, as indicated, is responsible for maintenance of the prophage state and generates superinfection immunity against homoimmune superinfecting phages. Induction occurs given the loss of the transcriptional repression necessary to sustain lysogeny, such as that mediated by CI. The result, given favorable conditions, is a productive infection, that is, maturation and then release of virion progeny (Figure 1).

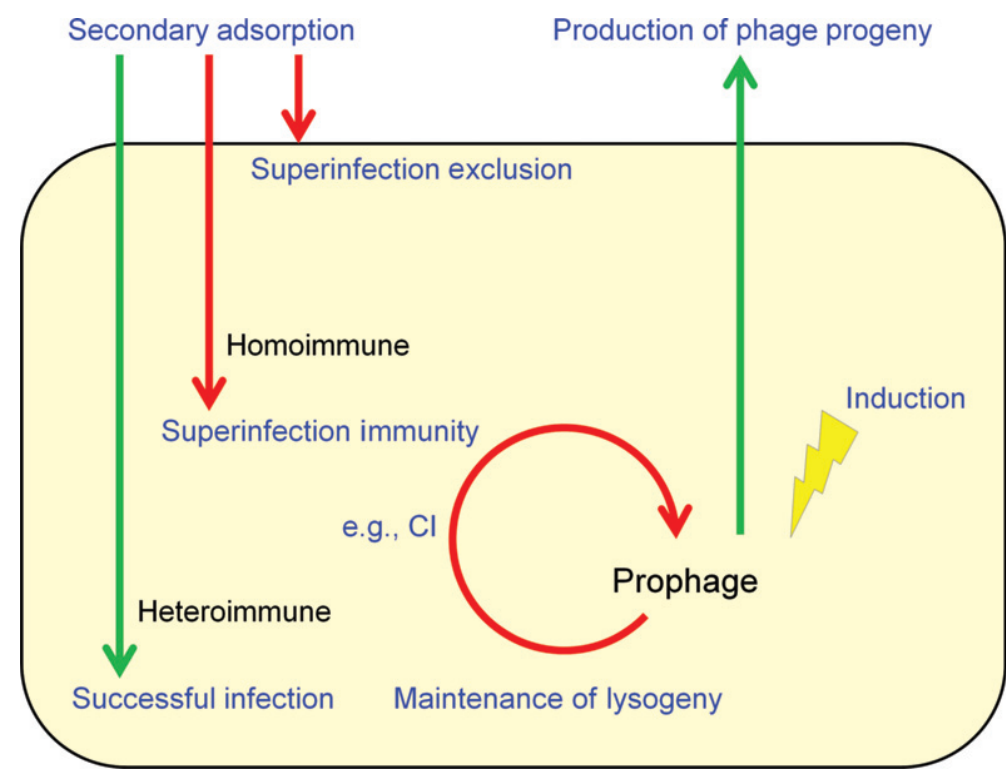

Figure 1 Overview of secondary adsorption, superinfection, superinfection immunity, and so on. 
See also: Analogy; Bacteria; Clever Mutant Isolation: Defining the Components of Cell Biological Processes; Fitness; Host-Range Mutant; Immunity: Phage, Plasmids, Transposons; Induction of Prophage; Lysis; Lysogeny; Mutation; Natural Selection; Operon; Phage (Bacteriophage); Phage $\lambda$ Integration/Excision; Plaques; Promoters; Prophage; Repressor; T Phages; Temperate Phage; Transcription; Wild Type.

\section{Further Reading}

Abedon ST (1999) Bacteriophage T4 resistance to lysis inhibition collapse. Genetical Beser 74: 1-11.

Abedon ST, Kuhl S, Blasdel B, and Kutter EM (2011) Phage treatment of human infections. Bacteriophage 1: 66-85

Campbell AM (2006) General aspects of lysogeny. In: Calendar R and Abedon ST (eds.) The Bacteriophages, 2nd edn. pp. 66-73. Oxford: Oxford University Press.

Dodd IB, Shearwin KE, and Egan JB (2005) Revisited gene regulation in phage $\lambda$. Current Opinion in Genetics \& Development 15: 145-152.

Fogg PCM, Rigden DJ, Saunders JR, McCarthy AJ, and Allison HE (2010) Characterization of the relationship between integrase, excisionase and antirepressof activities associated with a superinfecting Shiga toxin encoding bacteriophage. Nucleic Acids Research 39(6): 2116-2129.

Friedman DI and Court DL (2001) Bacteriophage lambda: Alive and well and still doing its thing. Current Opinion in Microbiology 4: 201-207.

Hendrix RW, Roberts JW, Stahl FW, and Weisberg RA (eds.) (1983) Lambda II. Cold Spring Harbor, NY: Cold Spring Harbor Laboratory Press.
Hershey AD (ed.) (1971) The Bacteriophage Lambda. Cold Spring Harbor, NY: Cold Spring Harbor Laboratory Press.

Hyman P and Abedon ST (2010) Bacteriophage host range and bacterial resistance. Advances in Applied Microbiology 70: 217-248.

Labrie SJ, Samson JE, and Moineau S (2010) Bacteriophage resistance mechanisms. Nature Reviews Microbiology 8: 317-327.

Little JW (2005) Lysogeny, prophage induction, and lysogenic conversion. In: Waldor MK, Friedman DI, and Adhya SL (eds.) Phages: Their Role in Bacterial Pathogenesis and Biotechnology, pp. 37-54. Washington DC: ASM Press.

Little JW (2006) Gene regulatory circuitry of phage $\lambda$. In: Calendar R and Abedon ST (eds.) The Bacteriophages, 2nd edn., pp. 74-82. Oxford: Oxford University Press.

Loc-Carrillo C and Abedon ST (2011) Pros and cons of phage therapy. Bacteriophage 1: $111-114$.

Meyer BJ, Kleid DG, and Ptashne M (1975) $\lambda$ Repressor turns off transcription of its own gene. Proceedings of the National Academy of Sciences of the United States of America 72: 4785-4789.

Ptashne M (2004) A Genetic Switch, 3rd edn. Cold Spring Harbor, NY: Cold Spring Harbor Laboratory Press.

\section{Relevant Websites}

http://books.google.com/books?id=hhrnA-t-sMkC - A genetic switch: phage lambda revisited.

http://www.phage.org/terms/immunity/ - Bacteriophage Ecology Group, immunity terms. http://splasho.com/blog/essays/bacteriophage-lambda/ - Bacteriophage lambda. http://www.landesbioscience.com/journals/bacteriophage/article/15845 LANDESBIOSCIENCE: Phage treatment of human infections.

http://www.landesbioscience.com/journals/bacteriophage/article/14590 LANDESBIOSCIENCE (Pros and cons of phage therapy). 